\title{
Mechanisms of autophagy and apoptosis mediated by JAK2 signaling pathway after spinal cord injury of rats
}

\author{
YONGZHI XIA, HAIJIAN XIA, DAN CHEN, ZHENGBU LIAO and YI YAN \\ Department of Neurosurgery, The First Affiliated Hospital of Chongqing Medical University, \\ Chongqing 400016, P.R. China
}

Received February 2, 2017; Accepted May 4, 2017

DOI: $10.3892 /$ etm.2017.4674

\begin{abstract}
The aim of this study was to investigate the pathogenesis of autophagy and apoptosis mediated by Janus kinase 2/signal transducer and activator of transcription 3 (JAK2/STAT3) signal pathway after the onset of acute spinal cord injury (ASCI). A total of 45 Sprague-Dawley adult rats of either sex were selected for this study. The age of rats ranged from 8 to 10 weeks, and the average weight was $245 \mathrm{~g}$. These rats were randomly divided into three groups, i.e. sham-operated group, model group, and the AG-490 intervention group (AG-490 is an inhibitor of JAK2). Each group contained 15 rats. Models were prepared using the modified Allen method. Five rats in each group were sacrificed at 6,12 and $24 \mathrm{~h}$, respectively, and the expression levels of p-JAK2 and p-STAT3 were detected in spinal cord tissue via western blot analysis. The levels of proinflammatory cytokines interleukin-6 (IL-6) and tumor necrosis factor- $\alpha(\mathrm{TNF}-\alpha)$ were detected via ELISA, positive expression of light chain 3 (LC3)-II of microtubule-associated protein 1 via immunofluorescence labeling method, and mRNA expression levels of caspase-3 and $\mathrm{Bax} / \mathrm{Bcl}-2$ via RT-PCR. In the model group, the expression levels of p-JAK2, p-STAT3, IL-6, TNF- $\alpha$ and LC3-II, and the mRNA expression levels of caspase- 3 and $\mathrm{Bax} / \mathrm{Bcl}-2$ at all time-points were significantly higher than those in the AG-490 intervention group, and the levels in the sham-operated group were the lowest $(\mathrm{p}<0.05)$. In the model group, peak levels of p-JAK2 and p-STAT3 were attained at $12 \mathrm{~h}$, but a decline was seen at $24 \mathrm{~h}$; while increasing trend was seen in other indicators. In conclusion, JAK2/STAT3 signal pathway can mediate the activity of autophagy and apoptosis in an early stage after the onset of ASCI of rat.
\end{abstract}

Correspondence to: Dr Yi Yan, Department of Neurosurgery, The First Affiliated Hospital of Chongqing Medical University, 1 You Yi Road, Chongqing 400016, P.R. China

E-mail: yanyi201601@163.com

Key words: JAK2/STAT3 signal pathway, acute spinal cord injury, autophagy, apoptosis

\section{Introduction}

Acute spinal cord injury (ASCI) can bring about mechanically structural damage and secondary neurological dysfunction, in which the loss of neuron may be an important cause for the permanent dysfunction of nerve after the onset of ASCI (1). Inflammatory response, cell autophagy and cell apoptosis are the important pathogeneses of neuron loss (2-4). Cell autophagy is a major method by which cells can maintain the survival, differentiation and homeostasis. In a study by Sekiguchi et al (5) it was reported that autophagy enhancement can alleviate the injury of spinal cord in rats, thus promoting the recovery of neurological functions. Another study from Erlich et al (6) confirmed that autophagy could exert the neuroprotective effect through inhibiting the already enhanced apoptosis. Peak level of spinal cord injury is usually attained at 24-48 $\mathrm{h}$ after the injury, which is coincident with the level of apoptosis in some tissue (7). In eukaryotes, JAK2/STAT3 signal pathway is a significant intracellular pathway, through which the expression of multiple cytokines and growth factors, cell proliferation, differentiation and apoptosis occurs $(8,9)$. In this study, we aimed to analyze the occurrences of autophagy and apoptosis mediated by JAK2/STAT3 signaling pathway after the ASCI of rats to provide a new target for intervention of ASCI at an early stage.

\section{Materials and methods}

ASCI model. We selected a total of 45 Sprague-Dawley adult rats of either sex. The age range of rats was from 8 to 10 weeks, and the average weight was $245 \mathrm{~g}$. These rats were purchased from the Experimental Animal Center of Sangon Biotech Co., Ltd. (Shanghai, China) and were prepared for study after 1 week of regular feeding. Modified Allen method was used to prepare the models through the following procedures. After $8 \mathrm{~h}$ of fasting, rats were anesthetized abdominally using $3 \%$ chloral hydrate $(27 \mathrm{mg} / 100 \mathrm{~g})$; rats were fixated in prone position, and an incision $(\sim 2.5 \mathrm{~cm}$ in length) was made in the middle of back; skin was cut layer by layer, and the T8 to T10 vertebral plates were exposed. Total laminectomy was performed for T9 vertebral plate to expose the dura mater spinalis; T8 and T10 spinous processes were fixated using forceps; Kirschner wire (10 g) was inserted into the catheter with scale, freely plummeted from $25 \mathrm{~mm}$ height, finally 
a semicircular slice ( $4 \mathrm{~mm}$ in diameter and $2 \mathrm{~mm}$ in width) made from thin plastic, was hit and the wire was immediately removed, leading to the incomplete injury of spinal cord in the rat; incision was sutured layer by layer. After the strike, rats with the signs of tail-wagging reflection, retraction flutter in lower limbs and body, and flaccid paralysis in lower limbs in awake state represented successful construction of the model. This study was approved by the Animal Ethics Committee of the First Affiliated Hospital of Chongqing Medical University.

Research method and observation indexes. These rats were randomly divided into three groups, i.e. sham-operated group, model group, and the AG-490 intervention group (AG-490 is an inhibitor of JAK2). Each group contained 15 rats. For rats in the sham-operated group, total laminectomy of T9 vertebral was only performed without any damage to spinal cord; for rats in the AG-490 intervention group, AG-490 (40 $\mu \mathrm{g} / \mathrm{g})$ was dissolved in $45 \%$ dimethyl sulfoxide (DMSO) and injected abdominally at $20 \mathrm{~min}$ before the spinal cord injury. Five rats in each group were sacrificed at 6,12 and $24 \mathrm{~h}$, respectively, and then we detected the p-JAK2 and p-STAT3 expression levels in spinal cord tissue via western blot analysis, and levels of interleukin-6 (IL-6) and tumor necrosis factor- $\alpha$ (TNF- $\alpha$ ) via enzyme-linked immunosorbent assay (ELISA), positive expression rate of light chain 3 (LC3)-II of microtubule-associated protein 1 via immunofluorescence labeling method, and mRNA expression levels of caspase-3 and $\mathrm{Bax} / \mathrm{Bcl}-2$ via RT-PCR.

\section{Detection method}

Western blot analysis. Excessive anesthesia was performed for rats using $3 \%$ chloral hydrate $(54 \mathrm{mg} / 100 \mathrm{~g})$. Thoracic cavity was opened, where the catheter was inserted into the aorta, and fixated using hemostatic forceps. Right auricle was incised, and the icy normal saline was rapidly perfused until the liquid turned clear. Then, the liquid was replaced by $4 \%$ paraformaldehyde for fixation for $40 \mathrm{~min}$. Spinal cord was stripped bluntly through the incision on the back, and we obtained the spinal cord $\sim 1 \mathrm{~cm}$ in length with the damaged part as the center. Spinal cord in $4 \mu \mathrm{m}$ of thickness was then acquired after fixation, dehydration, paraffin embedding and serial section. Spinal cord was then prepared into the tissue homogenate, in which the RIPA lysate was added for extraction of total protein of cells. Crude quantification was performed via Coomassie Brilliant Blue method, and the result was standardized using antibody for $\beta$-actin. Thirty micrograms of total protein was taken for separation via $8 \%$ sodium dodecyl sulfate-polyacrylamide gel electrophoresis (SDS-PAGE), and the separated strips were electrically transferred onto the PVDF membrane. Rabbit monoclonal JAK2 antibody (dilution, 1:500; cat. no. ab108596) and rabbit monoclonal STAT3 antibody (dilution, 1:500; cat. no. ab68153) were added onto the membrane for incubation overnight, and then secondary goat anti-rabbit (HRP) IgG antibody (dilution, 1:2,000; cat. no. an6721), was added onto the membrane for incubation for $4 \mathrm{~h}$ at room temperature. Thereafter, membrane was washed by phosphate-buffered saline (PBS) and colored using electrochemiluminescence (ECL). Results were scanned and preserved, and semi-quantitative analysis was carried out through Lab Works 4.5 gel imaging software (Invitrogen, Carlsbad, CA, USA) with the results being presented by integral optical density (IOD).
ELISA. After the tissue homogenate was prepared, centrifugation was performed at 2,500 x g for $30 \mathrm{~min}$. Supernatant was taken for later detection. Reagents were purchased from Jiangsu Beyotime Biotech Co., Ltd. (Jiangsu, China). All procedures were carried out in accordance with the manufacturer instructions.

Immunofluorescence labeling. Sections were blocked using normal goat serum for $1 \mathrm{~h}$. Then goat monoclonal LC3-II antibody (1:500; cat. no. TA803577; Beijing Zhongshan Golden Bridge Co., Ltd., Beijing, China) was added for incubation at $4^{\circ} \mathrm{C}$ overnight, and later immunofluorescence labeled rabbit anti-goat IgG secondary antibody (1:500; cat. no. ZDR-5308; Beijing Zhongshan Golden Bridge Co., Ltd.) was added onto the section for incubation at $4^{\circ} \mathrm{C}$ overnight. Sections were stained using 4',6-diamidino-2-phenylindole (DAPI) for 2 min, and sealed using glycerol after being washed. Then the section was placed under the fluorescence microscope for observation in the dark. In each group, we randomly selected 5 sections, and we chose upper, lower, left, right and central areas from the visions $(\mathrm{x} 400)$ to calculate the percentage of LC3-II positive cells.

$R T-P C R$. Total RNA in the cell was extracted using regular TRIzol reagent, and the concentration and purity were assayed through ultraviolet spectrometer. Reverse transcription kit was used for cDNA synthesis, and the primer sequences were synthesized by Sangon Biotech Co., Ltd. according to the sequences of Gene Bank: Bax forward, 5'-GGTTTCATCCA GGATCGAGCAGG-3' and reverse, 5'-ACAAAGATGGTCAC GGTCTGCC-3', 445 bp; Bcl-2 forward, 5'-ACTACTTCTCC CGCCGCTAC-3' and reverse, 5'-GAAATCAAACAGAGGCC GCATG-3', 332 bp; caspase-3 forward, 5'-TACCAGTGGAGG CCGACTTC-3' and reverse, 5'-GCACAAAGCGACTGGATG AAC-3', 103 bp; glyceraldehyde 3-phosphate dehydrogenase (GAPDH) forward, 5'-CGCGAGAAGATGACCCAGAT-3' and reverse, 5'-GCACTGTGTTGGCGTACAGG-3', 225 bp. Reaction system was set as follows: cDNA $2 \mu \mathrm{l}+$ upstream primer $3 \mu \mathrm{l}$ and downstream primer $3 \mu \mathrm{l}+$ Taq polymerase $0.5 \mu \mathrm{l}+\mathrm{dNTPs} 1 \mu \mathrm{l}+\mathrm{MgCl}_{2} 3 \mu \mathrm{l}+10 \mathrm{X}$ nuffer $5 \mu \mathrm{l}+\mathrm{ddH}_{2} \mathrm{O}_{2}$ $2.5 \mu \mathrm{l}$. Reaction conditions were set as follows: $95^{\circ} \mathrm{C}$ for $5 \mathrm{~min}$, $95^{\circ} \mathrm{C}$ for $30 \mathrm{sec}, 58^{\circ} \mathrm{C}$ for $30 \mathrm{sec}, 72^{\circ} \mathrm{C}$ for $60 \mathrm{sec}$ and for a total of 30 cycles followed by $72^{\circ} \mathrm{C}$ for $10 \mathrm{~min}$. PCR products were identified using $2 \%$ agarose gel electrophoresis. Ultraviolet images were developed through gel imaging analysis system. Grey value analysis was performed using the digital photographs. Results were analyzed by the $2^{-\Delta \Delta \mathrm{Cq}}$ method.

Statistical analysis. SPSS 20.0 (IBM, Armonk, NY, USA) was used for statistical analysis. Measurement data were presented by mean \pm standard deviation, single-factor ANOVA was performed for intergroup comparison, and LSD t-test for paired comparison; variance analysis of repeated measurements was used in the comparison among data in different time-points. $\mathrm{p}<0.05$ indicated that the difference had statistical significance.

\section{Results}

Expressions of $p$-JAK2 and p-STAT3. In the model group, expression levels of p-JAK2 and p-STAT3 at every time-point were significantly higher than those in the AG-490 intervention 

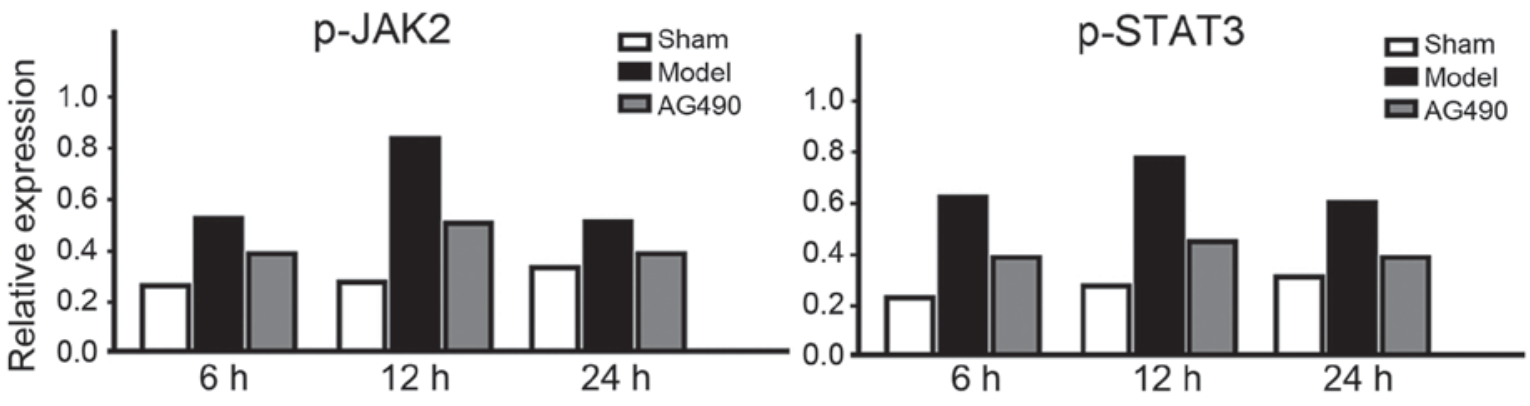

Figure 1. Expressions of p-Janus kinase 2 (JAK2) and p-signal transducer and activator of transcription 3 (STAT3).
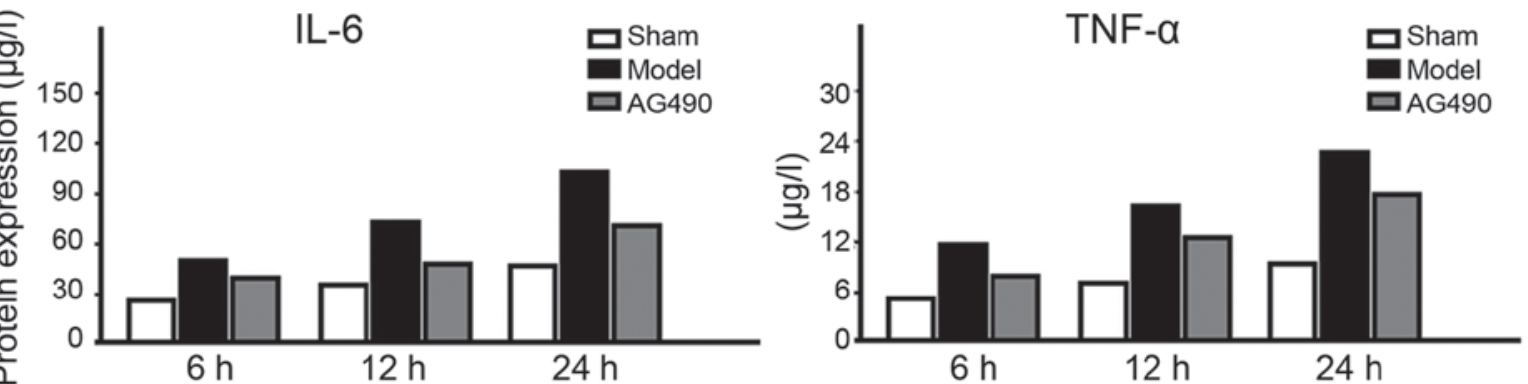

Figure 2. Levels of interleukin-6 (IL-6) and tumor necrosis factor- $\alpha$ (TNF- $\alpha$ ).

group, and the levels in the sham-operated group were the lowest $(\mathrm{p}<0.05)$. In the model group, peak levels of $\mathrm{p}-\mathrm{JAK} 2$ and p-STAT3 were attained at $12 \mathrm{~h}$, but a fall was seen at 24 h (Fig. 1).

Levels of IL- 6 and $T N F-\alpha$. In the model group, the expression levels of IL- 6 and TNF- $\alpha$ at every time-point were significantly higher than those in the AG-490 intervention group, and the levels in the sham-operated group were the lowest $(\mathrm{p}<0.05)$. In the model group, an increasing trend was seen in the expression levels of IL-6 and TNF- $\alpha$ (Fig. 2).

Positive expression of LC3-II. In the model group, positive expression rates of LC3-II at every time-point were significantly higher than those in the AG-490 intervention group, and the levels in the sham-operated group were the lowest $(\mathrm{p}<0.05)$. In the model group, an increasing trend was seen in the expression rates of LC3-II (Fig. 3).

mRNA expression levels of caspase-3 and Bax/Bcl-2. In the model group, mRNA expression levels of caspase-3 and Bax/Bcl-2 at every time-point were significantly higher than those in the AG-490 intervention group, and the levels in the sham-operated group were the lowest $(\mathrm{p}<0.05)$. In the model group, an increasing trend was seen in the mRNA expression levels of caspase-3 and Bax/Bcl-2 (Fig. 4).

\section{Discussion}

Under normal physiological condition, JAK2/STAT3 is found in non-phosphorylated condition; but when the body is under stress, phosphorylation of JAK2/STAT3 will be instantly completed, last for a short period, $\sim 6$ to $12 \mathrm{~h}$, and rapidly disappear (10). Through the intracellular binding site of tyrosine protein kinase, JAK2/STAT3 can bind with the relevant receptors, such as IL-6, TNF- $\alpha$, prolactin (PRL) and colony stimulating factor (CSF), thus activating the tyrosine residue of all downstream target proteins to exert the biological effect (11). In the study by Suzuki et al (12), it was found that IL- 6 can activate the JAK/STAT and p38-MAPK signal transduction pathways in the central nervous system to mediate the neurological injury and affect the neurological repair. Currently, it has been proven that JAK2/STAT3 signal pathway plays an important role in the immune reactions and the pathogenesis of tumor (13). The study by Wang et al (14) revealed that JAK2/STAT3 signal pathway can also induce apoptosis of the cortical neuron. As a substrate of JAK kinase, STAT, once being activated can pass through the nuclear membrane in the form of polymers, such as dimer or tetramer, to specifically bind with the response element on DNA to initiate the transcription of target downstream gene. Through this process, extracellular signals can be transduced into the cells to regulate various processes, such as cell proliferation, differentiation, apoptosis and immune regulations (15). STAT3 is a kind of bifunctional protein with key effect, and blocking JAK2/STAT3 signal pathway can significantly alleviate the focal cerebral ischemic re-perfusion injuries of rats, decrease the apoptosis of neurons, and ameliorate the impairment of neurologic functions (16).

Through this study, we found that in the model group, the expression levels of p-JAK2, p-STAT3, IL-6, TNF- $\alpha$ and LC3-II, and the mRNA expression levels of caspase-3 and $\mathrm{Bax} / \mathrm{Bcl}-2$ at every time-point were significantly higher than those in the AG-490 intervention group, and the levels in the sham-operated group were the lowest. In the model group, peak levels of p-JAK2 and p-STAT3 were attained at 

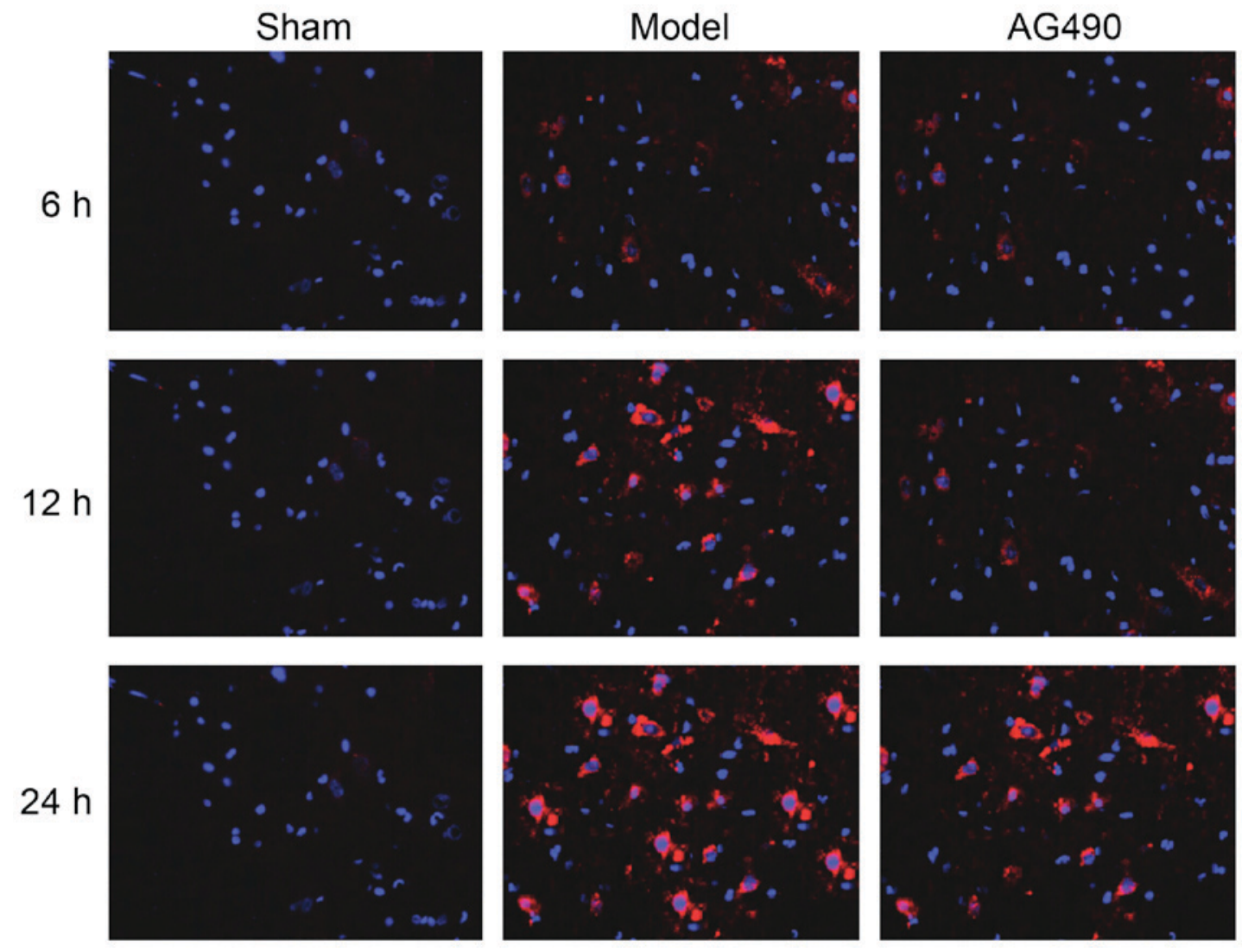

Figure 3. Positive expression of light chain 3 (LC3)-II (x400).
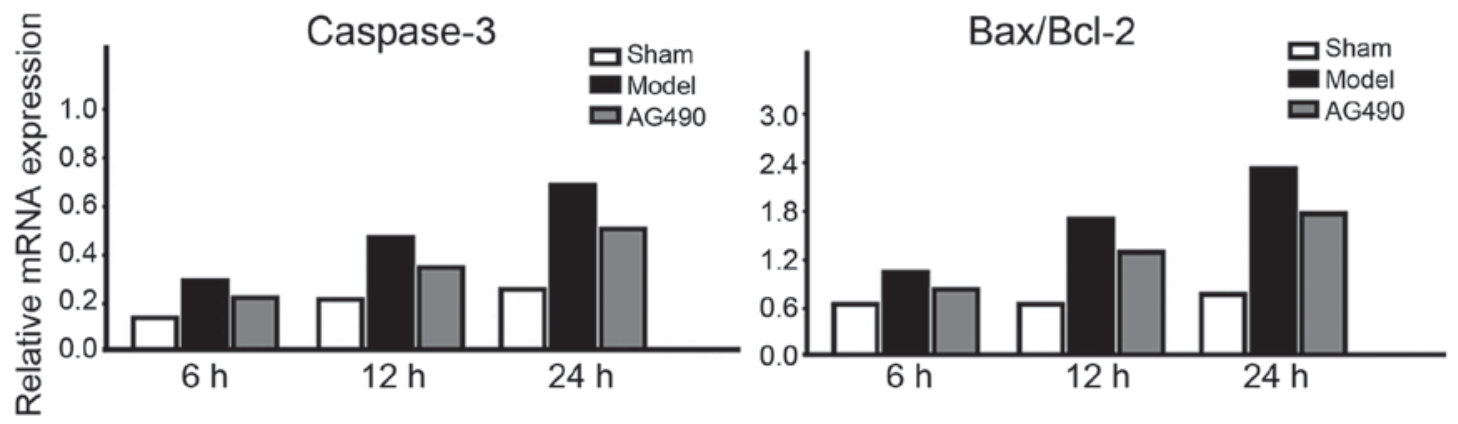

Figure 4. mRNA expression levels of caspase-3 and Bax/Bcl-2.

$12 \mathrm{~h}$, but a deline was seen at $24 \mathrm{~h}$; while increasing trend was seen in other indicators. This suggested that in an early stage, JAK2/STAT3 signal pathway can mediate autophagy and apoptosis activity after ASCI in rats. Activation of JAK2/STAT3 signal pathway requires the induction of inflammatory factors such as IL-6 and TNF- $\alpha$, which can in turn aggravate the release of inflammatory factors to induce the waterfall-like cascade reactions of inflammation to participate in the impairment of neurologic functions in the early and late stage of ASCI (17). Marker proteins that can reflect autophagy activity include LC3 and Atg12-Atg5 complex, in which LC3-I and LC3-II participate in the formation of autophagosome, combination between LC3-I and phosphatidylethanolamine can form LC3-II that is located on the membrane of autophagosome, and the content of LC3-II is positively correlated with the quantity of autophagosome (18). It was reported that the occurrence of autophagy is closely related with the apoptosis level, in which autophagy can inhibit the occurrence of excessively active apoptosis to promote the recovery of neurologic functions (19).

Different from cell necrosis, cell apoptosis refers to programmed cell death under precise regulation. Among various mitochondrion-dependent or non-mitochondrion dependent apoptosis mechanisms, apoptosis regulated by caspase family is a core member (20). Apoptosis is a cascade cleaving process of proteinase, and caspase-3, also known as the death proteinase, cleaving the protein kinase, nuclease and cytoskeleton, leading to a series of apoptotic activities, such as nuclear shrinkage condensation and DNA fragmentation, thus controlling the occurrence and progression of apoptosis (21). In addition, the orientation of apoptosis is generally decided by the ratio of $\mathrm{Bax}$ to $\mathrm{Bcl}-2$. If the ratio of $\mathrm{Bcl}-2$, the anti-apoptosis 
factor, to Bax, the pro-apoptosis factor, is not $<50 \%$, significant anti-apoptosis effect will emerge in the cells (22).

In conclusion, early intervention in autophagy, apoptosis and the key target point in the JAK2/STAT3 signal transduction pathway in ASCI may be of great significance for effectively alleviating the neurologic injuries and promoting recovery in neurologic functions.

\section{Acknowledgements}

This study was supported by the National Natural Science Foundation of China (no. 81100906) and the National Clinical Key Department Construction Programme of China (Cai She [2011]170).

\section{References}

1. Krause JS, Newman JC, Clark JM and Dunn M: The natural course of spinal cord injury: Changes over 40 years among those with exceptional survival. Spinal Cord 5: 123-124, 2016.

2. Kwan T, Floyd CL, Kim S and King PH: RNA binding protein $\mathrm{HuR}$ is translocated in astrocytes following spinal cord injury and promotes the inflammatory response. J Neurotrauma 6 : $125-126,2016$

3. Kanno H, Ozawa H, Sekiguchi A and Itoi E: Spinal cord injury induces upregulation of Beclin 1 and promotes autophagic cell death. Neurobiol Dis 33: 143-148, 2009.

4. Li G, Jia Z, Cao Y, Wang Y, Li H, Zhang Z, Bi J, Lv G and Fan Z: Mitochondrial division inhibitor 1 ameliorates mitochondrial injury, apoptosis, and motor dysfunction after acute spinal cord injury in rats. Neurochem Res 40: 1379-1392, 2015.

5. Sekiguchi A, Kanno H, Ozawa H, Yamaya $S$ and Itoi E: Rapamycin promotes autophagy and reduces neural tissue damage and locomotor impairment after spinal cord injury in mice. J Neurotrauma 29: 946-956, 2012.

6. Erlich S, Alexandrovich A, Shohami E and Pinkas-Kramarski R: Rapamycin is a neuroprotective treatment for traumatic brain injury. Neurobiol Dis 26: 86-93, 2007.

7. Wang S, Liu Y, Wu C, Zhao W, Zhang J, Bao G, Xu G, Sun Y, Chen J and Cui Z: The expression of IGFBP6 after spinal cord injury: Implications for neuronal apoptosis. Neurochem Res 5: 154-155, 2016.

8. Morales JK, Falanga YT, Depcrynski A, Fernando J and Ryan JJ: Mast cell homeostasis and the JAK-STAT pathway. Genes Immun 11: 599-608, 2010.

9. Lai SY and Johnson FM: Defining the role of the JAK-STAT pathway in head and neck and thoracic malignancies: Implications for future therapeutic approaches. Drug Resist Updat 13: 67-78, 2010.

10. Liu H, Yao YM, Yu Y, Dong N, Yin HN and Sheng ZY: Role of Janus kinase/signal transducer and activator of transcription pathway in regulation of expression and inflammation-promoting activity of high mobility group box protein 1 in rat peritoneal macrophages. Shock 27: 55-60, 2007.
11. Kang MK and Kang SK: Interleukin-6 induces proliferation in adult spinal cord-derived neural progenitors via the JAK2/STAT3 pathway with EGF-induced MAPK phosphorylation. Cell Prolif 41: 377-392, 2008.

12. Suzuki S, Tanaka K and Suzuki N: Ambivalent aspects of interleukin-6 in cerebral ischemia: Inflammatory versus neurotrophic aspects. J Cereb Blood Flow Metab 29: 464-479, 2009.

13. Kong LY, Abou-Ghazal MK, Wei J, Chakraborty A, Sun W, Qiao W, Fuller GN, Fokt I, Grimm EA, Schmittling RJ, et al: A novel inhibitor of signal transducers and activators of transcription 3 activation is efficacious against established central nervous system melanoma and inhibits regulatory T cells. Clin Cancer Res 14: 5759-5768, 2008.

14. Wang G, Zhou D, Wang C, Gao Y, Zhou Q, Qian G and DeCoster MA: Hypoxic preconditioning suppresses group III secreted phospholipase A2-induced apoptosis via JAK2-STAT3 activation in cortical neurons. J Neurochem 114: 1039-1048, 2010.

15. Xiong H, Zhang ZG, Tian XQ, Sun DF, Liang QC, Zhang YJ, Lu R, Chen YX and Fang JY: Inhibition of JAK1, 2/STAT3 signaling induces apoptosis, cell cycle arrest, and reduces tumor cell invasion in colorectal cancer cells. Neoplasia 10: 287-297, 2008.

16. Yang QZ, Lei C, Lu ZH, Wang BR and Xiong LZ: Neuroprotective effects of combined application of JAK-STAT signal pathway inhibitor and free radical scavenger on focal cerebral ischemia/reperfusion injury in rats. Zhongguo Wei Zhong Bing Ji Jiu Yi Xue 20: 641-644, 2008 (In Chinese).

17. Song Y, Zeng Z, Jin C, Zhang J, Ding B and Zhang F: Protective effect of ginkgolide $\mathrm{B}$ against acute spinal cord injury in rats and its correlation with the Jak/STAT signaling pathway. Neurochem Res 38: 610-619, 2013.

18. Hou H, Zhang L, Zhang L and Tang P: Acute spinal cord injury in rats should target activated autophagy. J Neurosurg Spine 20: 568-577, 2014.

19. Zhang YB, Li SX, Chen XP, Yang L, Zhang YG, Liu R and Tao LY: Autophagy is activated and may protect neurons from degeneration after traumatic brain injury. Neurosci Bull 24: $143-149,2008$.

20. Zhou Z, Chen S, Zhao H, Wang C, Gao K, Guo Y, Shen Z, Wang Y, Wang $\mathrm{H}$ and Mei X: Probucol inhibits neural cell apoptosis via inhibition of mTOR signaling pathway after spinal cord injury. Neuroscience 329: 193-200, 2016.

21. Gökce EC, Kahveci R, Gökce A, Cemil B, Aksoy N, Sargon MF, Kısa Ü, Erdoğan B, Güvenç Y, Alagöz F, et al: Neuroprotective effects of thymoquinone against spinal cord ischemia-reperfusion injury by attenuation of inflammation, oxidative stress, and apoptosis. J Neurosurg Spine 24: 949-959, 2016

22. Li CM, Xie SJ, Wang T, Du WB, Yang ZB and Quan RF: Effects of electro-acupuncture on neuronal apoptosis and associative function in rats with spinal cord injury. Zhongguo Gu Shang 28: 733-738, 2015 (In Chinese). 